\title{
Laser-Free GHz Stroboscopic TEM: Construction, Deployment, and Benchmarking
}

June W. Lau ${ }^{1 *}$, Karl B. Schliep ${ }^{1}$, Michael B. Katz ${ }^{1}$, Vikrant J. Gokhale ${ }^{1}$, and Jason J. Gorman ${ }^{1}$, Ao Liu ${ }^{2}$, Yubin $\mathrm{Zhao}^{2}$, Chunguang Jing ${ }^{2}$, Alexei Kanareykin ${ }^{2}$, Xuewen $\mathrm{Fu}^{3}$ and Yimei $\mathrm{Zhu}^{3}$

${ }^{1}$ National Institute of Standards and Technology, Gaithersburg, MD, USA.

2. Euclid TechLabs, 365 Remington Blvd., Bolingbrook, IL, USA.

3. Department of Condensed Matter Physics and Materials Science, Brookhaven National Laboratory, Upton, NY, USA.

*Corresponding author: june.lau@nist.gov

In the previous two decades, important technological advancements have expanded the range of temporal resolution in transmission electron microscopes (TEM). Commercial direct-counting and single-electron detectors have revealed dynamics in the ms-timescale. Laser-actuated photoemission microscopes $[1,2]$ combined with beam scanning, spatially-parsed large area detectors [3], and sparse-sensing algorithms [4], can now unlock phenomena at the $\mu$ s to sub-ps timescales. Further optimization of the photoemission stage [5] and beam bunching technologies could potentially extend the temporal resolution into the deep fs-regime.

Following our earlier concept paper [6], we now present the modifications to a pair of commercial instruments - one Schottky $(200 \mathrm{keV})$ and one thermionic $(300 \mathrm{keV})$ that can confer temporal information spanning the ns and ps range with $\mathrm{MHz}$ to $\mathrm{GHz}$ repetition rates, in the stroboscopic mode without an excitation laser. The key enabling technology is a pair of broadband phase-matched modulating and demodulating RF pulsers. Results shown in this abstract are from the $200 \mathrm{keV}$ Schottky instrument, demonstrating preliminary capabilities and core functionalities of this embodiment of the concept. The minimum guaranteed temporal resolution of $192 \mathrm{ps}$ and $100 \mathrm{ps}$ were achieved with strobe frequencies of $2.6 \mathrm{GHz}$ and $5.0 \mathrm{GHz}$ respectively (FIG. 1). The placement of the pulsers, mounted immediately below the gun, allows for the preservation of all optical configurations otherwise available to the unmodified instrument, and therefore makes these instruments dual-mode, both stroboscopic time-resolved (strobe) mode and conventional continuous waveform $(\mathrm{CW})$ mode.

To show that the modifications preserved imaging and diffraction functionalities of the instrument, we obtained Au images and diffraction patterns using both a continuous and a strobed beam at $2.6 \mathrm{GHz}$ (FIG. 2). As the probe portion of our "pump-probe" system is nearing completion, this is a good occasion to discuss the technical challenges associated with the pump portion - the science of RF transmission to the specimen [7].

References:

[1] VA Lobastov, R Srinivasan and AH. Zewail, Proc. Natl. Acad. Sci. 102 (2005), p. 7069.

[3] T LaGrange et. al., Appl. Phys. Lett. 89 (2006), p. 044105.

[3] T LaGrange, BW Reed and DJ. Masiel, MRS Bulletin 40 (2015), p. 22.

[4] A Stevens et al., Advanced Structural and Chemical Imaging 1 (2015).

[5] DA Plemmons and DJ Flannigan, Chemical Physics Letters 683 (2017), p. 186.

[6] J Qiu et. al., Ultramicroscopy 161 (2016), p. 130. 
[7] We are grateful for the invaluable assistance from JEOL USA. This work was supported by DOE BES SBIR program Grant no. DE-SC0013121 and NIST award SB1341-16-CN-0035. During this work, K. Schliep was supported by the Research Associateship Program of the National Research Council and M. Katz was partially supported by the Professional Research Experience Program at the University of Maryland. Work at BNL was supported by the US DOE-BES, MSED, under Contract No.

DESC0012704.
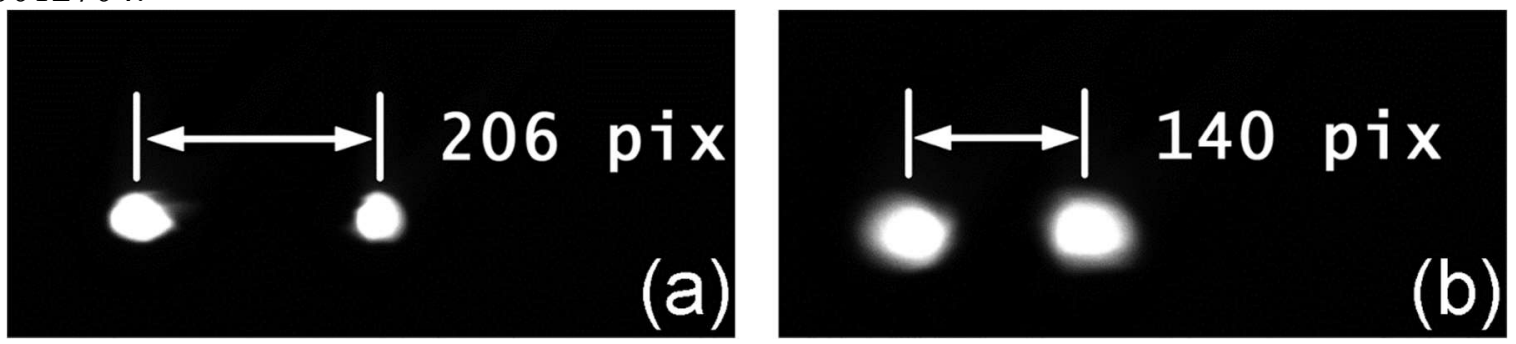

Figure 1. First experimental demonstration of temporal resolution displayed as a split beam image. In normal strobe mode, the two beams are coincident. However, in the enhanced modulation diagnostic mode, the downstream demodulation pulser was deliberately de-tuned with respect to the upstream modulation pulser, causing the split-beam. The magnitude of the split is an accurate proxy for the magnitude of RF modulation, which is inversely proportional to the minimum guaranteed temporal resolution. In (a) and (b), the measured minimum temporal resolution was $192 \mathrm{ps}(2.6 \mathrm{GHz}$ input) and $100 \mathrm{ps}(5.0 \mathrm{GHz})$, respectively. Modulator at $0.49 \mathrm{~V}$, demodulator at $0.1 \mathrm{~V}$ for both cases. Insertion of a beam shopping aperture produces the final temporal resolution, nominally at $10 \%$ beam duty cycle, or $19.2 \mathrm{ps}$ and $10.0 \mathrm{ps}$ for the two strobe frequencies shown.
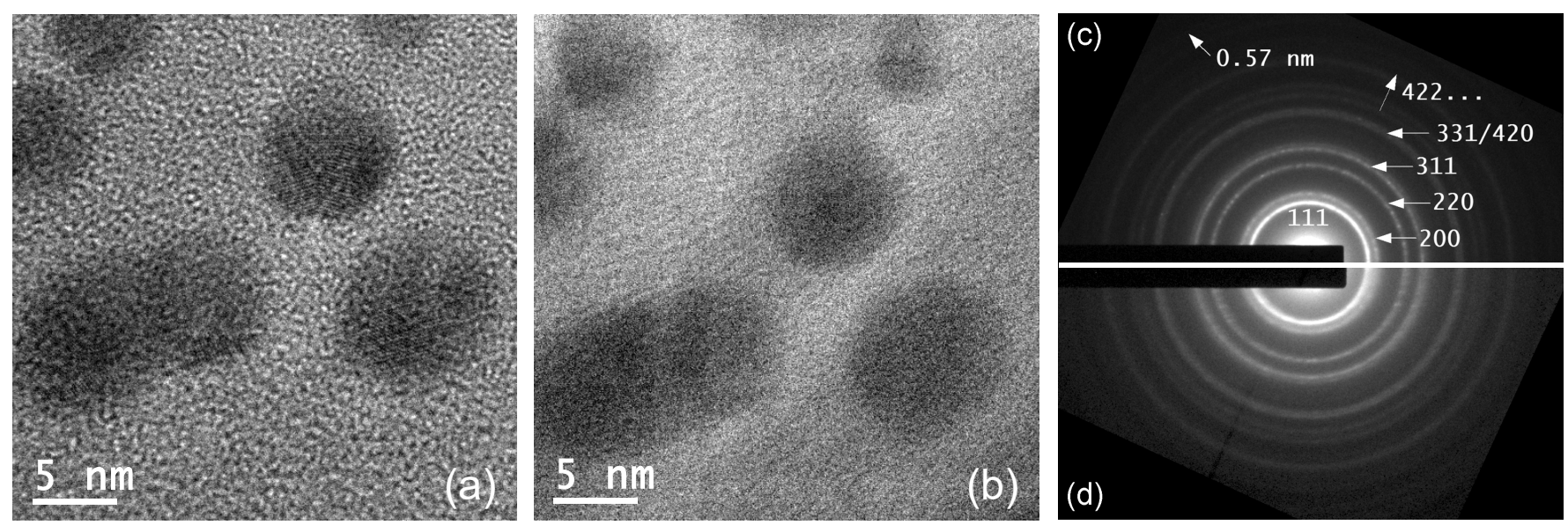

Figure 2. Image and diffraction quality comparison between $\mathrm{CW}$ and strobe modes using a $\mathrm{Au}$ nanoparticles sample. A $2.6 \mathrm{GHz}$ input was used to produce images and diffraction patterns obtained in the strobe mode. In (a), indicated magnification of $600 \mathrm{kX}$ with a $\mathrm{CW}$ beam was used. Lattice fringes are visible, thus demonstrating that high-(spatial) resolution imaging mode of this instrument is preserved with this modification. (b) The same condition as (a) but with a strobed beam. Image quality optimization in the strobe mode is a project goal for this year. (c) A reference selected area diffraction pattern with an indicated camera length of $20 \mathrm{~cm}$. The last ring on this micrograph at $0.57 \mathrm{~nm}$ can be readily resolved. (d) same condition as (c) but with a strobed beam. Ring appearance is less sharp than the reference, but higher spatial frequencies are also preserved. 